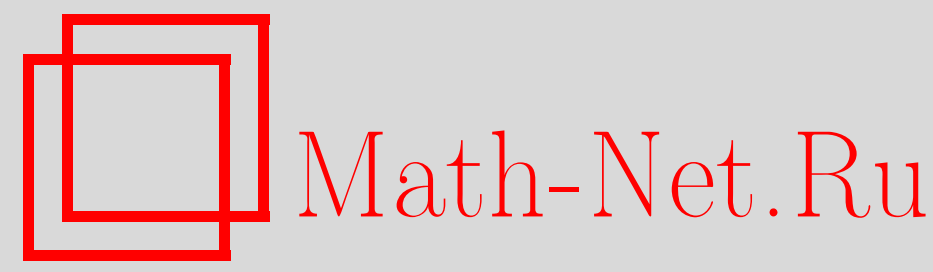

И. В. Аржанцев, О. В. Чувашова, Классификация аффинных однородных пространств сложности один, Матем. сб., 2004, том 195, номер 6, 3-20

DOI: https://doi.org/10.4213/sm819

Использование Общероссийского математического портала Math-Net.Ru подразумевает, что вы прочитали и согласны с пользовательским соглашением http://www.mathnet.ru/rus/agreement

Параметры загрузки:

IP: 54.81 .137 .203

26 апреля 2023 г., 15:14:21 
УДК 512.745

\author{
И.В. Аржанцев, О.В. Чувашова
}

\title{
Классификация аффинных однородных пространств сложности один
}

\begin{abstract}
Сложностью действия редуктивной алгебраической группы $G$ на многообразии $X$ называют коразмерность типичной орбиты для индуцированного действия борелевской подгрупшы $B \subset G$ на $X$. В работе классифицированы афффинные однородные пространства $G / H$ сложности один. Эти результаты являются естественньм продолжением полученной ранее классификации сфферических афффинных однородных пространств, т.е. пространств сложности нуль.
\end{abstract}

Библиографиия: 21 название.

\section{§1. Введение}

Пусть $G$ - связная редуктивная алгебраическая группа над алгебраически замкнутьм полем $\mathbb{K}$ нулевой характеристики и $H$ - алгебраическая подгруппа в $G$. С однородньм пространством $G / H$ связан важный целочисленный инвариант $c(G / H)$ - его сложность. Понятие сложности однородного пространства возникло в работе [1], а для произвольного $G$-многообразия сложность была введена в [2]. Как показали исследования последующих десятилетий, сложность играет ключевую роль при изучении геометрии однородного пространства, в теории его эквивариантных вложений, в теории инвариантных гамильтоновых систем на кокасательном расслоении $T^{*}(G / H)$ и в других разделах математики, связанных с однородными пространствами.

Напомним определение сложности. Пусть алгебраическая группа $G$ действует на неприводимом алгебраическом многообразии $X$ и $B$ - борелевская подгруппа в $G$. Сложностью $c_{G}(X) G$-многообразия $X$ называют минимальную коразмерность $B$-орбиты на $X$ для индуцированного действия $B: X$. (Мы будем обозначать сложность через $c(X)$, когда это не может привести к недоразумениям.) По теореме Розенлихта сложность равна степени трансцендентности поля $\mathbb{K}(X)^{B}$ рациональных $B$-инвариантных функций на $X$. Кроме сложности имеется еще одна важная характеристика действия - его ранг. Рангом $\operatorname{rk}(X)$ неприводимого $G$-многообразия $X$ называют ранг решетки весов $B$-полуинвариантных рациональных функций на $X$.

Нормальное $G$-многообразие $X$ назьвают сферическим, если $c(X)=0$, или, эквивалентно, $\mathbb{K}(X)^{B}=\mathbb{K}$. Пространство $G / H$ и подгруппа $H \subseteq G$ называются

Работа выполнена при финансовой поддержке Российского фонда фундаментальных исследований (грант № 01-01-00756), МАС (гранты № № 02-01-06401, 03-01-06252) и гранта Президента РФ (№ MK-1279.2004.1).

(С) И. В. АРЖАНцев, О. В. ЧувАШОвА 2004 
сферическими, если $G / H$ является сферическим $G$-многообразием. Теория сферических многообразий является одним из наиболее разработанных разделов теории алгебраических групп преобразований (см., например, обзор [3]). В частности, для сферических однородных пространств построена замечательная теория вложений [1], [4], обобщающая теорию торических многообразий. В работе [5] получено аналогичное (но существенно более сложное) описание вложений однородных пространств сложности один.

Пространства малой сложности естественно возникают при изучении вопроса о конечности числа орбит во вложениях данного однородного пространства. Так, в произвольном вложении сферического однородного пространства число орбит конечно, в то время как любое однородное пространство сложности большей или равной 1 допускает проективное вложение с бесконечным числом орбит [6]. Более обшо, наибольшее значение $G$-модальности (т.е. максимума числа параметров в непрерьвном семействе $G$-орбит) по всем вложениям пространства $G / H$ равно $c(G / H)[2],[7]$.

Если ограничиться аффинньми однородньми пространствами и их вложениями в аффинные многообразия, то известно, что каждое аффинное вложение пространства $G / H$ сложности большей или равной 2 содержит лишш конечное число орбит тогда и только тогда, когда $G / H$ аффинно замкнуто, т.е. каждое аффинное вложение $G / H$ состоит ровно из одной орбиты [8]. Аффинное однородное пространство сложности один допускает аффинное вложение с бесконечным числом орбит тогда и только тогда, когда подгруппа $H$ может быть расширена одномерным централизуюшим ее тором так, что сложность получившегося однородного пространства остается равной единице [8]. Среди однородных пространств простых групп этим свойством обладают только пространства, отвечающие п. 3 таблицы 2 настояшей работы. В случае полупростой группы таких пространств сушественно больше они соответствуют пп. $9,10,15,17$ и 26 таблицы 4 . О связи сложности и модальности афффинных вложений см. [9].

Другой причиной интереса к однородным пространствам малой сложности служат задачи симплектической геометрии и теории интегрируемых гамильтоновых систем. Пусть $G$ - связная вешественная редуктивная группа Ли и $K$ - ее связная замкнутая редуктивная подгруппа. Кокасательное расслоение $T^{*}(G / K)$ несет естественную структуру симплектического многообразия, и действие $G: G / K$ левыми сдвигами индуцирует симплектическое действие $G: T^{*}(G / K)$. Обозначим буквой $P$ связанное с этим действием отображение моментов $P: T^{*}(G / K) \rightarrow \mathfrak{g}^{*}$. Алгебры вешественно-аналитических функций $C\left(T^{*}(G / K)\right)$ и $C\left(\mathfrak{g}^{*}\right)$ канонически наделяются скобками Пуассона, причем для любых $h_{1}, h_{2} \in C\left(\mathfrak{g}^{*}\right)$ имеем

$$
\left\{h_{1} \circ P, h_{2} \circ P\right\}=\left\{h_{1}, h_{2}\right\} \circ P .
$$

Функции на $T^{*}(G / K)$ вида $h \circ P$ назьвают коллективнылми. По теореме Нётер коллективные функции являются интегралами для любого потока на $T^{*}(G / K)$ с $G$-инвариантньм гамильтонианом. Другими словами, $\{h \circ P, f\}=0$ для любой $f \in C\left(T^{*}(G / K)\right)^{G}$. Говорят, что $G$-инвариантная гамильтонова система $\dot{x}=\operatorname{sgrad} f$ интегрируема в классе интегралов Нётер, или коллективно вполне интегрируема, если найдется набор $\left\{h_{i}\right\}, i=1, \ldots, \operatorname{dim} G / K$, вешествен- 
но-аналитических функций на $\mathfrak{g}^{*}$ такой, что функции $h_{i} \circ P$ функционально независимы и находятся попарно в инволюции. Известно, что всякая $G$-инвариантная гамильтонова система на $T^{*}(G / K)$ интегрируема в классе интегралов Нётер тогда и только тогда, когда комплексифицированное однородное пространство $G^{\mathbb{C}} / K^{\mathbb{C}}$ сферично [10]. Если подгруппа $K$ компактна, то указанные условия эквивалентны обращению в нуль скобки Пуассона на любой паре функций из $C\left(T^{*}(G / K)\right)^{G}[11]$ (см. также [12; предложение 9]).

Исследованию свойств гамильтоновых систем на кокасательных расслоениях однородных пространств положительной сложности посвящены работы [13], [14]. Там показано, что для компактной подгруппы $K$ число независимых $G$-инвариантных вещественно-аналитических функций на $T^{*}(G / K)$, которые независимы также от коллективных функций, равно $2 c\left(G^{\mathbb{C}} / K^{\mathbb{C}}\right)$. (В работе [12] доказано, что это же число равно корангу симплектического действия $G^{\mathbb{C}}: T^{*}\left(G^{\mathbb{C}} / K^{\mathbb{C}}\right)$.) В свою очередь, максимальное число независимых коллективных функций в инволюции равно $\operatorname{dim} G / K-c\left(G^{\mathbb{C}} / K^{\mathbb{C}}\right)$. В случае однородных пространств сложности один ${ }^{1}$, добавив к максимальной независимой системе коллективных функций в инволюции независимую от них инвариантную функцию $F$, мы получим полную систему функций в инволюции. Выбирая $F$ коммутирующей с гамильтонианом, можно показать, что на кокасательном расслоении к однородному пространству сложности один любая $G$-инвариантная гамильтонова система интегрируема $[14$; предложение 12].

Таким образом, однородные пространства малой сложности редуктивной группы $G$ являются весьма естественньм и важным для приложений объектом. Поэтому хотелось бы иметь полный список таких пространств $G / H$. Классификацию естественно начать со случая, когда подгруппа $H$ редуктивна. (Согласно критерию Мацусимы это условие эквивалентно аффинности пространства $G / H$.) При таком ограничении списки однородных пространств сложности меньшей или равной 1 имеют разумный объем. Это подтверждает предположение, высказанное в конце работы [2], где задача классификации пространств сложности один была поставлена впервые.

Хорошо известно, что сложность пространства $G / H$ определяется парой касательных алгебр $(\mathfrak{g}, \mathfrak{h})$ (предложение 1$)$. Это позволяет свести классификацию к перечислению пар $(\mathfrak{g}, \mathfrak{h})$, где $\mathfrak{g}$ - редуктивная алгебра Ли, $\mathfrak{h}$ - ее редуктивная подалгебра, для которых типичная коразмерность в алгебре $\mathfrak{g}$ подпространств вида $\mathfrak{h}+\operatorname{Ad}(g) \mathfrak{b}, g \in G$, равна единице. Без ограничения обшности можно считать алгебру $\mathfrak{g}$ полупростой (предложение 2 ).

Цель настоящей работы - классифицировать все аффинные однородные пространства сложности один редуктивных групп в терминах касательных алгебр. Результаты классификации приведены в таблице 4. Ранее была получена классификация сферических редуктивных подгрупп в простых группах [15], классификация сферических редуктивных подгрупп в произвольных редуктивных группах (см. [10] и, независимо, [16]), а также классификация редуктивных подгрупп сложности один в простых группах [17] (см. также [13]). Для удобства читателя результаты этих классификаций включены в текст работы (см. таблицы 1, 3 и 2 соответ-

\footnotetext{
${ }^{1}$ В работе [14] они названы почти сферическими (almost spherical spaces).
} 
ственно). Отметим, что классификация проводится с точностью до автоморфизма объемлющей алгебры $\mathfrak{g}$. Так, сферические пары $\left(\mathfrak{s o}_{8}, \mathfrak{s p i n}_{7}\right)$ и $\left(\mathfrak{s o}_{8}, \mathfrak{s p}_{4} \oplus \mathfrak{s l}_{2}\right)$ переводятся внешним автоморфизмом алгебры $\mathfrak{s o}_{8}$ в пары, отвечающие п. 10 таблицы 1 при $(n, m)=(7,1)$ и $(5,3)$ соответственно, поэтому отдельно в таблице 1 не фигурируют. В таблицах 1 и 2 мы указали также $\mathfrak{h}$-модуль $\mathfrak{m}$, отвечающий представлению изотропии. В таблицах 3 и 4 представление изотропии не указано, однако его легко найти, поскольку пары из этих таблиц получены описанной ниже операцией сцепки пар из таблиц 1 и 2 , после которой представление изотропии есть прямая сумма представлений изотропии сцепливаемых пар и одного экземпляра присоединенного представления той компоненты, по которой проводилась сцепка. В таблицах 3 и 4 указаны стационарные подалгебры общего положения для представления изотропии и ранги соответствующих однородных пространств (заметим, что ранг пространства $G / H$ также определяется парой $(\mathfrak{g}, \mathfrak{h})$, см. [19]).

Использованный нами метод классификации близок к методу работы И. В. Микитюка [10]. В частности, операция, которую мы называем сцепкой, в [10] называлась "расширением пар". Отметим, что использование операции сцепки двух пар алгебра-подалгебра позволяет избежать рассмотрения глубины подалгебры (М. Брион) и проводить индукцию лишш по числу простых компонент объемлющей алгебры g. Вычисление сложности однородного пространства основано на изучении стационарной подалгебры обшего положения для представления изотропии (здесь используются таблицы Элашвили [18]) и применении формул Панюшева (теоремы 1 и 2 ).

Авторы благодарны Д. А. Тимашёву, который обратил их внимание на принципиальную возможность полученной в настоящей работе классификации, а также сделал ряд ценных замечаний.

\section{§ 2. Необходимые сведения о сложности и ранге однородных пространств}

Всюду в тексте алгебры Ли алгебраических групп будем обозначать соответствующими строчньми готическими буквами. Исключение сделано только для особых простых алгебр Ли - по традиции они обозначены большими латинскими буквами. В этом параграфе мы приведем некоторые хорошо известные факты о сложности однородных пространств.

Лемма 1. Пусть $F$ - редуктивная подгруппа в $G$ и $H$ - алгебраическая nодгруппа в $F$. Тогда

1) $c_{G}(G / F) \leqslant c_{G}(G / H)$;

2) $c_{F}(F / H) \leqslant c_{G}(G / H)$.

ДоказАТЕЛЬСтво. Согласно [2] сложность $G$-многообразия $X$ равна модальности для индуцированного действия $B$ на $X$. В случае, когда $X$ есть однородное пространство $G / H$, указанная модальность совпадает с модальностью двустороннего действия $B \times H$ на $G$ и правого действия $H$ на $B \backslash G$. Отсюда следует неравенство 1). Эти же соображения доказывают 2), если выбрать борелевскую $B$ в $G$ так, чтобы пересечение $B$ и $F$ было борелевской подгруппой $B_{F}$ в $F$, и реализовать $B_{F} \backslash F$ как $F$-орбиту точки $B e$ в $B \backslash G$. 
ПРЕДЛОЖЕНИЕ 1. Следующие условия әквивалентны:

1) $c(G / H)=m$;

2) существует открытое подмнохсество $W \subset G$ такое, что для любого $g \in W \quad \operatorname{codim}_{\mathfrak{g}}(\mathfrak{h}+\operatorname{Ad}(g) \mathfrak{b})=m$.

ДокАЗАТЕльство. Условие 1) эквивалентно тому, что для точек $g$ из открытого подмножества $V \subset G$ подмножество $B g H$ (или $g^{-1} B g H$ ) имеет в $G$ коразмерность $m$. Последнее эквивалентно условию 2) для $W=V^{-1}$.

Будем говорить, что пара $(\mathfrak{g}, \mathfrak{h})$ имеет сложность $m$, если выполнено условие 2). Далее для сложности пары $(\mathfrak{g}, \mathfrak{h})$ используем обозначение $c(\mathfrak{g}, \mathfrak{h})$. Предложение 1 позволяет свести классификацию однородных пространств сложности один к классификации соответствующих пар $(\mathfrak{g}, \mathfrak{h})$.

ПРЕДЛОЖЕНИЕ 2. Пусть $\mathfrak{g}=\mathfrak{g}^{s} \oplus \mathfrak{z}-$ разложение редуктивной алгебрь $\mathfrak{g}$ в сумму максимального полупростого идеала и иентра. Тогда сложсность пары $(\mathfrak{g}, \mathfrak{h})$ равна сложсности пары $\left(\mathfrak{g}^{s}, \operatorname{pr}_{1}(\mathfrak{h})\right)$, әде $\operatorname{pr}_{1}: \mathfrak{g} \rightarrow \mathfrak{g}^{s}-$ проекция вдоль $\mathfrak{z}$.

ДоказАтЕльство. Нужно заметить, что $\mathfrak{z}$ лежит в $\operatorname{Ad}(g) \mathfrak{b}$ для всех $g \in G$. Поэтому $\mathfrak{h}+\operatorname{Ad}(g) \mathfrak{b}=\operatorname{pr}_{1}(\mathfrak{h})+\operatorname{Ad}(g) \mathfrak{b}$.

Предложение 2 позволяет считать алгебру $\mathfrak{g}$ полупростой. Всюду далее подгруппа $H$ и подалгебра $\mathfrak{h}$ предполагаются редуктивными.

Рассмотрим ограничение присоединенного представления $\mathrm{Ad}: G \rightarrow \mathrm{GL}(\mathfrak{g})$ на подгруппу $H$. Подалгебра $\mathfrak{h}$ является инвариантным подпространством для такого представления и в силу редуктивности $H$ в $\mathfrak{g}$ можно найти $H$-инвариантное подпространство $\mathfrak{m}$, дополнительное к $\mathfrak{h}$. Заметим, что $H$-модуль $\mathfrak{m}$ может быть отождествлен с касательным пространством в точке $e H$ к аффинному однородному пространству $G / H$. Линейное представление $\rho: H \rightarrow \mathrm{GL}(\mathfrak{m})$ называют представлением изотропии для пары $(G, H)$. Его дифференциал $d \rho: \mathfrak{h} \rightarrow \mathfrak{g l}(\mathfrak{m})$ однозначно определяется парой $(\mathfrak{g}, \mathfrak{h})$. Для представления $d \rho$ обозначим через $\mathfrak{s}$ стационарную подалгебру общего положения (с.п.о.п.). Известно, что s редуктивна [19; теорема 3]. Пусть $\mathfrak{b}$ - подалгебра Бореля в $\mathfrak{g}, \mathfrak{b}_{\mathfrak{s}}-$ подалгебра Бореля в $\mathfrak{s}$ и $N(\mathfrak{g})$ - число положительных корней в системе корней, отвечающей алгебре $\mathfrak{g}$. Следуюшая теорема показывает, в частности, что ранг пространства $G / H$ определяется парой $(\mathfrak{g}, \mathfrak{h})$, что оправдывает обозначение $\operatorname{rk}(\mathfrak{g}, \mathfrak{h})$.

Теорема 1 [19; теорема 3]. Имеют место соотношения:

$$
\begin{gathered}
c(G / H)=c(\mathfrak{g}, \mathfrak{h})=\operatorname{dim} \mathfrak{b}-\operatorname{rk} \mathfrak{g}-\operatorname{dim} \mathfrak{h}+\operatorname{dim} \mathfrak{b}_{\mathfrak{s}}=N(\mathfrak{g})-\operatorname{dim} \mathfrak{h}+N(s)+\operatorname{rk} \mathfrak{s} \\
\operatorname{rk}(G / H)=\operatorname{rk}(\mathfrak{g}, \mathfrak{h})=\operatorname{rkg}-\operatorname{rk} \mathfrak{s} .
\end{gathered}
$$

Также для наших вычислений будет нужна

Теорема 2 [20; теорема 1.2]. Предположим, что подалгебра $\mathfrak{h}$ вложена в подалгебру Леви $\mathfrak{l}$ параболической подалгебры $\mathfrak{p}$ алгебры $\mathfrak{g}$ u $S_{1}$ - стабилизатор общего положсения для действия $H$ на факторе l/h. Тогда

$$
c(\mathfrak{g}, \mathfrak{h})=c(\mathfrak{l}, \mathfrak{h})+c_{S_{1}}\left(\mathfrak{p}^{u}\right),
$$


где $\mathfrak{p}^{u}$ - унипотентный радикал алгебры $\mathfrak{p}$. В частности, если $c(\mathfrak{l}, \mathfrak{h})=0$, то

$$
c(\mathfrak{g}, \mathfrak{h})=\operatorname{dim} \mathfrak{p}^{u}-\operatorname{dim} \mathfrak{b}_{\mathfrak{s}_{1}}+\operatorname{dim} \mathfrak{b}_{1},
$$

əде $\mathfrak{b}_{1}-$ c.n.o.n. для действия $\mathfrak{b}_{\mathfrak{s}_{1}}$ на $\mathfrak{p}^{u}$.

\section{§3. Редуктивные подалгебры полупростых алгебр Ли}

Пусть $\mathfrak{g}=\mathfrak{g}_{1} \oplus \cdots \oplus \mathfrak{g}_{s}-$ разложение редуктивной алгебры Ли $\mathfrak{g}$ в прямую сумму простых идеалов. Будем называть $\mathfrak{g}_{i}$ компонентами алгебры $\mathfrak{g}$. Для полупростой $\mathfrak{g}$ все $\mathfrak{g}_{i}$ являются простьми (некоммутативными) алгебрами Ли, а для редуктивной $\mathfrak{g}$ некоторые из $\mathfrak{g}_{i}$ могут быть одномерны.

Пусть $\mathfrak{h}$ - редуктивная подалгебра в $\mathfrak{g}$ и $\mathfrak{h}(i)$ - проекция $\mathfrak{h}$ на $\mathfrak{g}_{i}$ вдоль остальных компонент. Тогда $\mathfrak{h}(i)$ - редуктивная подалгебра в $\mathfrak{g}_{i}$. Будем говорить, что подалгебра $\mathfrak{h}$ вполне разложима, если $\mathfrak{h}=\mathfrak{h}(1) \oplus \cdots \oplus \mathfrak{h}(s)$. В обшем случае $\mathfrak{h}$ содержится во вполне разложимой подалгебре $\mathfrak{h}^{r}=\mathfrak{h}(1) \oplus \cdots \oplus \mathfrak{h}(s)$ и $\mathfrak{h}^{r}$ - минимальная вполне разложимая подалгебра, содержашая $\mathfrak{h}$.

Из сказанного следует, что описание редуктивных подалгебр в $\mathfrak{g}$ состоит из двух этапов: описание вполне разложимых подалгебр (что сводится к описанию редуктивных подалгебр в простых алгебрах) и описание редуктивных подалгебр, для которых проекция на каждую компоненту алгебры сюръективна.

Лемма 2. Пусть $\mathfrak{g}=\mathfrak{g}_{1} \oplus \cdots \oplus \mathfrak{g}_{s}-$ полупростая алгебра Ли $и \mathfrak{h}-$ редуктивная подалгебра в $\mathfrak{g}$, для которой проекция на каждуую компоненту $\mathfrak{g}_{i}$ сюрвективна. Тогда $\mathfrak{h}$ полупроста и любая ее компонента $\mathfrak{h}_{j}$ вкладшвается в $\mathfrak{g}_{1} \oplus \cdots \oplus \mathfrak{g}_{s}$ как $x \mapsto\left(\operatorname{pr}_{j 1}(x), \ldots, \operatorname{pr}_{j s}(x)\right)$, где $\operatorname{pr}_{j i}: \mathfrak{h}_{j} \rightarrow \mathfrak{g}_{i}-$ либо изоморфизм, либо нулевое отображение (т.е. $\mathfrak{h}_{j}$ диагонально вкладывается в произведение некоторых изоморфных междуу собой $\mathfrak{g}_{i}$ ). Для фиксированного $i$ ровно одно отображение $\mathrm{pr}_{j i}$ отлично от нуля.

ДокАЗАТЕЛЬСтво. Из сюръективности проекции $\operatorname{pr}_{i}: \mathfrak{h} \rightarrow \mathfrak{g}_{i}$ следует, что идеалы $\mathfrak{h}$ переходят в идеалы $\mathfrak{g}_{i}$. В силу простоты алгебры $\mathfrak{g}_{i}$ образ любого $\mathfrak{h}_{j}$ есть либо 0 , либо $\mathfrak{g}_{i}$. Отсюда следует, что в $\mathfrak{h}$ нет коммутативных идеалов (т.е. $\mathfrak{h}$ полупроста). Более того, во втором случае из простоты $\mathfrak{h}_{j}$ следует, что $\mathrm{pr}_{j i}-$ изоморфизм. Наконец, ядро проекции $\mathrm{pr}_{i}: \mathfrak{h} \rightarrow \mathfrak{g}_{i}-$ это идеал в $\mathfrak{h}$, и, значит, он есть сумма некоторых компонент алгебры $\mathfrak{h}$. Тем самым $\mathrm{pr}_{i}$ определяет изоморфизм $\mathfrak{g}_{i}$ и суммы тех компонент $\mathfrak{h}_{j}$, которые не попали в ядро $\mathrm{pr}_{i}$. Это доказывает последнее утверждение леммы.

Нетрудно обобщить лемму 2 на случай редуктивной g. В этой ситуации центры $\mathfrak{g}$ и $\mathfrak{h}$ будут совпадать.

Пусть $\mathfrak{v}=\mathfrak{v}_{1} \oplus \cdots \oplus \mathfrak{v}_{t}-$ редуктивная алгебра Ли, и пусть $\varphi_{i, j}: \mathfrak{v}_{i} \rightarrow \mathfrak{v}_{j}-$ изоморфизм между некоторьми компонентами. Будем говорить, что подалгебра

$$
\mathfrak{h}=\left\{\left(x_{1}, \ldots, x_{i}, \ldots, x_{j-1}, \varphi_{i j}\left(x_{i}\right), x_{j+1}, \ldots, x_{t}\right) \mid x_{i} \in \mathfrak{v}_{i}, i \neq j\right\}
$$

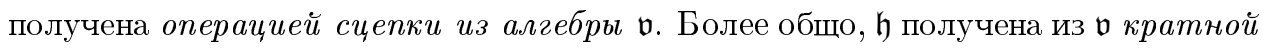
cцепкой, если она получена из $\mathfrak{v}$ последовательным применением (конечного числа) сцепок. 
Вывод. Каждая редуктивная подалгебра $\mathfrak{h}$ полупростой алгебры Ли

$$
\mathfrak{g}=\mathfrak{g}_{1} \oplus \cdots \oplus \mathfrak{g}_{s}
$$

может быть получена из вполне разложимой подалгебры $\mathfrak{w}=\mathfrak{w}(1) \oplus \cdots \oplus \mathfrak{w}(s)$ $\left(=\mathfrak{h}^{r}\right)$, где $\mathfrak{w}(i)$ - редуктивные подалгебры в $\mathfrak{g}_{i}$, кратной сцепкой некоторых компонент алгебры w.

\section{§4. Метод классификации}

Будем говорить, что пара $(\mathfrak{g}, \mathfrak{h})$ разложима, если найдется нетривиальное разложение $\mathfrak{g}$ в прямую сумму идеалов $\mathfrak{g}^{1}$ и $\mathfrak{g}^{2}$, для которого $\mathfrak{h}=\mathfrak{h}^{1} \oplus \mathfrak{h}^{2}$, где $\mathfrak{h}^{i}=$ $\mathfrak{g}^{i} \cap \mathfrak{h}$. Из предложения 1 следует, что для разложимой подалгебры $\mathfrak{h}=\mathfrak{h}^{1} \oplus \mathfrak{h}^{2}$ в $\mathfrak{g}=\mathfrak{g}^{1} \oplus \mathfrak{g}^{2}$ сложность аддитивна: $c(\mathfrak{g}, \mathfrak{h})=c\left(\mathfrak{g}^{1}, \mathfrak{h}^{1}\right)+c\left(\mathfrak{g}^{2}, \mathfrak{h}^{2}\right)$. Это позволяет в классификации ограничиться только неразложимьми парами.

Будем вести классификацию, используя индукцию по числу $s$ компонент алгебры g. При $s=1$ алгебра $\mathfrak{g}$ проста, и все пары сложности 0 (соответственно 1) собраны в таблице 1 (соответственно таблице 2). Предположим, мы нашли все пары $(\mathfrak{g}, \mathfrak{h})$ сложности меньшей или равной 1 для всех алгебр $\mathfrak{g}$, у которых $s=k$ $($ список $k$ ). Тогда мы найдем все интересующие нас пары $(\mathfrak{g}, \mathfrak{h})$ для $s=k+1$, производя кратную сцепку в подалгебре $\mathfrak{h}^{1} \oplus \mathfrak{h}^{2}$, где $\left(\mathfrak{g}^{1}, \mathfrak{h}^{1}\right)$ - пара из списка $k$, а пара $\left(\mathfrak{g}^{2}, \mathfrak{h}^{2}\right)$ содержится в таблице 1 или 2. При этом хотя бы одна из пар $\left(\mathfrak{g}^{1}, \mathfrak{h}^{1}\right)$ или $\left(\mathfrak{g}^{2}, \mathfrak{h}^{2}\right)$ должна быть сферической (из первого пункта леммы 1 следует, что $\left.c(\mathfrak{g}, \mathfrak{h}) \geqslant c\left(\mathfrak{g}^{1}, \mathfrak{h}^{1}\right)+c\left(\mathfrak{g}^{2}, \mathfrak{h}^{2}\right)\right)$. Заметим также, что в $\mathfrak{h}^{1} \oplus \mathfrak{h}^{2}$ достаточно рассматривать сцепки только между простыми компонентами, лежащими в разных слагаемых.

Пару $(\mathfrak{g}, \mathfrak{h})$ будем называть насыщенной, если центр алгебры $\mathfrak{h}$ - вполне разложимая подалгебра в $\mathfrak{g}$ (в работе [10] использовался термин “главные пары”). Это соответствует тому, что $\mathfrak{h}$ получена из вполне разложимой подалгебры алгебры $\mathfrak{g}$ сцепками неодномерных компонент. Мы классифицируем насышенные неразложимые пары сложности один. Отсюда будет следовать и классификация всех неразложимых пар (см. замечание 2 ниже).

Пусть $(\mathfrak{g}, \mathfrak{h})$ - пара сложности меньшей или равной 1 и $\mathfrak{h}_{i}-$ компонента $\mathfrak{h}$. Будем говорить, что $\mathfrak{h}_{i}$ валентна (соответственно 1-валентна), если $\mathfrak{h}_{i}$ не является одномерной и подалгебра, полученная сцепкой по компоненте $\mathfrak{h}_{i}$ в подалгебре $\mathfrak{h} \oplus \mathfrak{h}_{i}$, сферична в $\mathfrak{g} \oplus \mathfrak{h}_{i}($ соответственно имеет сложность 1$)$. Указанную сцепку мы будем называть әлементарной.

Понятие валентности позволяет существенно сократить число сцепок, подлежащих рассмотрению. А именно, при неэлементарной сцепке все искомые пары получаются сцепкой двух валентных компонент или валентной и 1-валентной компоненты (используя теорему 1 , несложно показать, что сложность прямой суммы двух элементарных сцепок $\left(\mathfrak{g}_{1} \oplus \mathfrak{h} \oplus \mathfrak{g}_{2} \oplus \mathfrak{h}, \mathfrak{h}_{1} \oplus \mathfrak{h} \oplus \mathfrak{h}_{2} \oplus \mathfrak{h}\right)$ не больше сложности неэлементарной сцепки $\left.\left(\mathfrak{g}_{1} \oplus \mathfrak{g}_{2}, \mathfrak{h}_{1} \oplus \mathfrak{h} \oplus \mathfrak{h}_{2}\right)\right)$ и дальнейшей кратной сцепкой в полученных алгебрах.

Этап 1. Начнем с того, что определим все валентные и 1-валентные компоненты для пар $(\mathfrak{g}, \mathfrak{h})$, где $\mathfrak{g}$ проста. Из определения сложности вытекает, что

$$
c(\mathfrak{g}, \mathfrak{h}) \geqslant N(\mathfrak{g})-\operatorname{dim} \mathfrak{h} .
$$


Отсюда для валентности (соответственно 1 -валентности) компоненты $\mathfrak{h}_{i}$ необходимо, чтобы $N(\mathfrak{g})+N\left(\mathfrak{h}_{i}\right)-\operatorname{dim} \mathfrak{h} \leqslant 0$ (соответственно меньше или равно 1$)$. При помощи этого условия из пар, перечисленных в таблицах 1 и 2 , мы получаем следующий список кандидатов на валентные или 1-валентные компоненты (они подчеркнуты):

$$
\begin{aligned}
& \left(\mathfrak{s l}_{3}, \underline{\mathfrak{s O}_{3}}\right), \quad\left(\mathfrak{s l}_{n+m}, \underline{\mathfrak{s l}_{m}} \oplus \mathfrak{s l}_{n} \oplus \mathfrak{c}\right)^{2}, \quad\left(\mathfrak{s l}_{n+m}, \underline{\mathfrak{s l}_{m}} \oplus \mathfrak{s l}_{n}\right)^{3}, \\
& \left(\mathfrak{s o}_{n+m}, \underline{\mathfrak{s o}_{m}} \oplus \mathfrak{s o}_{n}\right)^{4}, \quad\left(\mathfrak{s o}_{7}, \underline{G_{2}}\right), \quad\left(\mathfrak{s p}_{4}, \underline{\mathfrak{s l}_{2}} \oplus \mathfrak{c}\right)^{5}, \quad\left(\mathfrak{s p}_{2(n+m)}, \underline{\mathfrak{s p}_{2 m}} \oplus \mathfrak{s p}_{2 n}\right)^{6}, \\
& \left(G_{2}, \underline{\mathfrak{s l}_{3}}\right), \quad\left(G_{2}, \underline{\mathfrak{s l}_{2}} \oplus \mathfrak{s l}_{2}\right), \quad\left(F_{4}, \underline{\mathfrak{s l}_{2}} \oplus \mathfrak{s p}_{6}\right), \quad\left(E_{6}, \underline{\mathfrak{s l}_{2}} \oplus \mathfrak{s l}_{6}\right), \\
& \left(E_{7}, \underline{\mathfrak{s l}_{2}} \oplus \mathfrak{s o}_{12}\right), \quad\left(E_{8}, \underline{\mathfrak{s l}_{2}} \oplus E_{7}\right), \quad\left(\mathfrak{s p}_{2 n}, \underline{\mathfrak{s l}_{2}} \oplus \mathfrak{s l}_{2} \oplus \mathfrak{s p}_{2 n-4}\right) .
\end{aligned}
$$

Для того чтобы выяснить, какие из этих компонент в самом деле валентны или 1-валентны, воспользуемся теоремами 1 и 2.

ЗАмечание 1. Рассмотрим пары $\left(\mathfrak{g}_{1}, \mathfrak{s l}_{2} \oplus \mathfrak{h}_{1}\right)\left(\mathfrak{s}_{1}-\right.$ с.п.о.п. представления изотропии, сложность равна $\left.c_{1}\right),\left(\mathfrak{g}_{2}, \mathfrak{s l}_{2} \oplus \mathfrak{h}_{2}\right)\left(\mathfrak{s}_{2}-\right.$ c.п.о.п., сложность равна $\left.c_{2}\right)$ и сцепку подалгебры по компоненте $\mathfrak{s l}_{2}:\left(\mathfrak{g}_{1} \oplus \mathfrak{g}_{2}, \mathfrak{s l}_{2} \oplus \mathfrak{h}_{1} \oplus \mathfrak{h}_{2}\right)(\mathfrak{s}-$ с.п.о.п., сложность равна $c$ ). Из теоремы 1 вытекает, что $c=c_{1}+c_{2}$ в точности тогда, когда в одной из пар $(i=1,2)$ проекция $\mathfrak{s}_{i}$ на компоненту $\mathfrak{s l}_{2}$ сюръективна, а в другой паре аналогичная проекция ненулевая. Условие $c=c_{1}+c_{2}+1$ равносильно тому, что либо одна из проекций сюръективна, а другая нулевая, либо обе проекции одномерны (их образами служат подалгебры Картана в $\mathfrak{s l}_{2}$ ). В остальных случаях $c \geqslant c_{1}+c_{2}+2$. У алгебры 5 проекция на компоненту $\mathfrak{s l}_{2}$ нулевая за исключением одного случая - и в $\mathfrak{s}_{1}$, и в $\mathfrak{s}_{2}$ проекции на компоненту $\mathfrak{s l}_{2}$ сюръективны. В последнем случае проекция $\mathfrak{s}$ на компоненту $\mathfrak{s l}_{2}$ одномерна.

Из замечания 1 следует, что при элементарной сцепке по компоненте $\mathfrak{s l}_{2}$ сложность возрастает не более чем на единицу и $\mathfrak{s l}_{2}$-компонента сферической пары всегда валентна или 1-валентна (в первом случае пара содержится в таблице 3 ). Методом исключения из сферических пар получаем 1-валентные компоненты:

$$
\begin{gathered}
\left(\mathfrak{s l}_{3}, \underline{\mathfrak{s o}_{3}}\right), \quad\left(G_{2}, \underline{\mathfrak{s l}_{2}} \oplus \mathfrak{s l}_{2}\right), \quad\left(F_{4}, \underline{\mathfrak{s l}_{2}} \oplus \mathfrak{s p}_{6}\right), \quad\left(E_{6}, \underline{\mathfrak{s l}_{2}} \oplus \mathfrak{s l}_{6}\right), \\
\left(E_{7}, \underline{\mathfrak{s l}_{2}} \oplus \mathfrak{s o}_{12}\right), \quad\left(E_{8}, \underline{\mathfrak{s l}_{2}} \oplus E_{7}\right), \quad\left(\mathfrak{s p}_{4}, \underline{\mathfrak{s l}_{2}} \oplus \mathfrak{c}\right), \quad\left(\mathfrak{s l}_{3}, \underline{\mathfrak{s l}_{2}}\right), \quad\left(\mathfrak{s o}_{n+3}, \underline{\mathfrak{s o}_{3}} \oplus \mathfrak{s o}_{n}\right) .
\end{gathered}
$$

Нетрудно показать, что кандидаты, возникшие из пар сложности один, доставляют 1-валентные компоненты:

$$
\left(\mathfrak{s p}_{2 n}, \underline{\mathfrak{s l}}_{2} \oplus \mathfrak{s l}_{2} \oplus \mathfrak{s p}_{2 n-4}\right), \quad\left(\mathfrak{s l}_{4}, \underline{\mathfrak{s l}_{2}} \oplus \mathfrak{s l}_{2}\right) .
$$

Перейдем к вопросу о валентности компонент, отличных от $\mathfrak{s l}_{2}$. Применим теорему 2 к сцепкам $\left(\mathfrak{s l}_{n+m} \oplus \mathfrak{s l}_{m}, \underline{\mathfrak{s l}_{m}} \oplus \mathfrak{s l}_{n} \oplus \mathfrak{c}\right)$ и $\left(\mathfrak{s l}_{n+m} \oplus \mathfrak{s l}_{m}, \underline{\mathfrak{s l}_{m}} \oplus \mathfrak{s l}_{n}\right)$. В первом

\footnotetext{
${ }^{2}$ При некоторых условиях на $m, n$.

${ }^{3}$ При некоторых условиях на $m, n$.

${ }^{4}$ При некоторых условиях на $m, n$.

${ }^{5}$ Вложение соответствует строке 17 таблицы 1 .

${ }^{6}$ При некоторых условиях на $m, n$.
} 
случае $\mathfrak{b}_{1}=\mathfrak{c} \oplus \mathfrak{b}^{n-m}$ (здесь $\mathfrak{b}^{n-m}$ - борелевская подалгебра в $\mathfrak{s l}_{n-m}$ ) при $m<n$ и $\mathfrak{b}_{1}=0$ при $m \geqslant n$. Во втором случае $\mathfrak{b}_{1}=\mathfrak{b}^{n-m}$ при $m<n$ и $\mathfrak{b}_{1}=0$ при $m \geqslant n$. Получаем валентные компоненты:

$$
\left(\mathfrak{s l}_{n+2}, \underline{\mathfrak{s l}_{2}} \oplus \mathfrak{s l}_{n} \oplus \mathfrak{c}\right), \quad\left(\mathfrak{s l}_{m+1}, \underline{\mathfrak{s l}_{m}} \oplus \mathfrak{c}\right), \quad\left(\mathfrak{s l}_{n+2}, \underline{\mathfrak{s l}_{2}} \oplus \mathfrak{s l}_{n}, n \geq 3\right)
$$

и 1-валентные компоненты

$\left(\mathfrak{s l}_{n+3}, \underline{\mathfrak{s l}_{3}} \oplus \mathfrak{s l}_{n} \oplus \mathfrak{c}, n \geq 2\right), \quad\left(\mathfrak{s l}_{n+3}, \underline{\mathfrak{s l}_{3}} \oplus \mathfrak{s l}_{n}, n \geq 4\right), \quad\left(\mathfrak{s l}_{m+1}, \underline{\mathfrak{s l}_{m}}\right), \quad\left(\mathfrak{s l}_{4}, \underline{\mathfrak{s l}_{2}} \oplus \mathfrak{s l}_{2}\right)$.

Рассмотрим пару $\left(\mathfrak{s o}_{n+m} \oplus \mathfrak{s o}_{m}, \mathfrak{s o}_{m} \oplus \mathfrak{s o}_{n}\right)$. Здесь представление изотропии имеет вид $m=\pi_{1} \otimes \pi_{1}^{\prime} \oplus \operatorname{ad}\left(\mathfrak{s o}_{m}\right)$. Отсюда, $\mathfrak{s}=\mathfrak{s o}_{n-m} \subset \mathfrak{s o}_{n}$ при $n>m$ и $\mathfrak{s}=0$ при $n \leqslant m$. Таким образом, получаем валентную компоненту $\left(\mathfrak{s o}_{m+1}, \underline{\mathfrak{s o}_{m}}\right)$ и 1-валентную компоненту $\left(\mathfrak{s o}_{n+3}, \underline{\mathfrak{s o}}_{3} \oplus \mathfrak{s o}_{n}\right)$.

Рассмотрим пару $\left(\mathfrak{s p}_{2(n+m)} \oplus \overline{\mathfrak{s p}_{2 m}}, \underline{\mathfrak{s p}_{2 m}} \oplus \mathfrak{s p}_{2 n}\right)$. Представление изотропии имеет вид $m=\pi_{1} \otimes \pi_{1}^{\prime} \oplus \operatorname{ad}\left(\mathfrak{s p}_{2 m}\right)$. Получаем $\mathfrak{s}=\mathfrak{c} \oplus \mathfrak{s p}_{2 n-2}$ при $m=1$ и $\mathfrak{s}=\mathfrak{s p}_{2(n-m)} \subseteq \mathfrak{s p}_{2 n}$ при $m \geqslant 2(\mathfrak{s}=0$ при $n \leqslant m)$. Отсюда, имеем валентные компоненты $\left(\mathfrak{s p}_{2(n+2)}, \underline{\mathfrak{s p}_{4}} \oplus \mathfrak{s p}_{2 n}\right),\left(\mathfrak{s p}_{2(n+1)}, \underline{\mathfrak{s p}_{2}} \oplus \mathfrak{s p}_{2 n}\right)$ и 1-валентную компоненту $\left(\mathfrak{s p}_{8}, \mathfrak{s p}_{6} \oplus \mathfrak{s p}_{2}\right)$.

В сцепке $\left(\mathfrak{s o}_{7} \oplus G_{2}, \underline{G_{2}}\right)$ представление изотропии имеет вид $m=\pi_{1} \oplus \operatorname{ad}\left(G_{2}\right)$. Здесь $\mathfrak{s}=0$ и компонента 1-валентна.

Рассмотрим пару $\left(G_{2} \oplus \mathfrak{s l}_{3}, \underline{\mathfrak{s l}}_{3}\right)$. Здесь $\mathfrak{s l}_{3}$ вложена в $G_{2}$ "по длинным корням", следовательно, короткие корни линейно независимы на подалгебре Картана в $\mathfrak{s l}_{3}$, $\mathfrak{s}=0$ и компонента 1-валентна.

Тем самым найдены все валентные и 1-валентные простые компоненты для пар $(\mathfrak{g}, \mathfrak{h})$ сложности меньшей или равной 1, где $\mathfrak{g}$ проста.

Этап 2. Сейчас нам будет удобно несколько отступить от стандартного ведения индукции по параметру $s$. Мы укажем все неразложимые насышенные пары сложности один, полученные из пар, отвечающих простьм алгебрам, сцепками по компонентам, изоморфным $\mathfrak{s l}_{2}$.

Из замечания 1 и списка всех валентных и 1-валентных компонент, изоморфных $\mathfrak{s l}_{2}$ (или из явного вида представления изотропии), следует, что во всех парах списка кандидатов (этап 1), где подалгебра $\mathfrak{h}$ имеет компоненту, изоморфную $\mathfrak{l}_{2}$, проекция на нее подалгебры $\mathfrak{s}$ нулевая за исключением пар

1) $\left(\mathfrak{s l}_{n+2}, \mathfrak{s l}_{2} \oplus \mathfrak{s l}_{n} \oplus \mathfrak{c}\right), n \geqslant 1,\left(\mathfrak{s l}_{n+2}, \mathfrak{s l}_{2} \oplus \mathfrak{s l}_{n}\right), n \geqslant 2,\left(\mathfrak{s p}_{2 n}, \mathfrak{s l}_{2} \oplus \mathfrak{s l}_{2} \oplus \mathfrak{s p}_{2 n-4}\right)$, $n \geqslant 3,-$ здесь проекция одномерна;

2) $\left(\mathfrak{s p}_{2(n+1)}, \mathfrak{s l}_{2} \oplus \mathfrak{s p}_{2 n}\right)$ - здесь проекция сюръективна.

Замечание 1 и только что указанная информация о проекциях позволяют найти все пары сложности 1 , полученные из пар $(\mathfrak{g}, \mathfrak{h})$ с простой $\mathfrak{g}$ сцепками по компонентам $\mathfrak{s l}_{2},-$ получаем пш. 1-22 таблицы 4 .

Этап 3. Несложные вычисления, основанные на соображениях размерности и явном виде представления изотропии, показывают, что все неэлементарные сцепки по валентньм и 1-валентным компонентам, отличным от $\mathfrak{s l}_{2}$, приводят к парам сложности больше единицы.

В заключение вносим в таблищу 4 пп. 24-29, отвечающие найденным ранее 1-валентньм компонентам. 
Этап 4. Перейдем к перечислению валентных и 1-валентных компонент, отличных от $\mathfrak{s l}_{2}$, в парах сложности меньшей или равной 1 при $s=2$. Здесь нужно рассматривать лишь те компоненты, которые были валентны либо 1-валентны в парах, отвечающих простым алгебрам, сцепкой которых была получена данная пара.

Начнем с п. 9 таблицы 3 . Компонента $\mathfrak{h}$ в этой паре 1-валентна тогда и только тогда, когда $N(\mathfrak{h})-\mathrm{rk} \mathfrak{h}=1$, а это имеет место только для $\mathfrak{h}=\mathfrak{s l}_{3}$ (получаем п. 30 таблицы 4). Из п. 2) леммы 1 следует, что в паре сложности один никакая компонента $\mathfrak{h}$, кроме изоморфных $\mathfrak{s l}_{2}$ или $\mathfrak{s l}_{3}$, не может вкладываться более чем в две простые компоненты алгебры $\mathfrak{g}$.

Остается проверить на валентность либо 1-валентность подчеркнутые компоненты в парах следующего типа:

$$
\begin{array}{ll}
\left(\mathfrak{g}_{1} \oplus \mathfrak{s p}_{2 n+4}, \mathfrak{h}_{1} \oplus \mathfrak{s p}_{2 n} \oplus \underline{\mathfrak{s p}_{4}}\right), & n=1,2, \\
\left(\mathfrak{g}_{1} \oplus \mathfrak{s p}_{8}, \mathfrak{h}_{1} \oplus \mathfrak{s l}_{2} \oplus \underline{\mathfrak{s p}_{6}}\right), & \\
\left(\mathfrak{g}_{1} \oplus \mathfrak{s l}_{n+3}, \mathfrak{h}_{1} \oplus \mathfrak{s l}_{n} \oplus \underline{\mathfrak{s l}_{3}}\right), & n=2,3, \\
\left(\mathfrak{g}_{1} \oplus \mathfrak{s l}_{n+3}, \mathfrak{h}_{1} \oplus \mathfrak{g l}_{n} \oplus \underline{\mathfrak{s l}_{3}}\right), & n=2,3, \\
\left(\mathfrak{g}_{1} \oplus \mathfrak{g}_{2}, \mathfrak{h}_{1} \oplus \underline{\mathfrak{s l}_{3}} \oplus \mathfrak{h}_{2}\right), &
\end{array}
$$

где первая компонента $\mathfrak{h}$ вложена только в $\mathfrak{g}_{1}$, третья - только во вторую компоненту $\mathfrak{g}$, а вторая - в обе компоненты $\mathfrak{g}$.

Теорема 1 показывает, что для валентности либо 1-валентности подчеркнутой компоненты необходимо, чтобы проекция на нее алгебры $\mathfrak{s}$ для указанной пары содержала борелевскую подалгебру размерности не менее $3,8,2,2$ и 2 соответственно. Используя явньй вид подалгебры $\mathfrak{s}$, вычисленный для всех пар таблицы 4 при $s=2$, получаем, что это условие вьполнено только для $\left(\mathfrak{s p}_{2 n+2} \oplus \mathfrak{s p}_{6}, \mathfrak{s p}_{2 n} \oplus\right.$ $\mathfrak{s l}_{2} \oplus \mathfrak{s p}_{4}$ ). Нетрудно показать, что компонента $\mathfrak{s p}_{4}$ здесь 1-валентна (таблица 4 , п. 23).

Этап 5. Нетривиальную сцепку для $s=3$ по компоненте, отличной от $\mathfrak{s l}_{2}$, имеет смысл рассматривать только между п. 16 таблицы 1 при $m=2$ и п. 3 таблицы 3 при $m=2$ по компоненте $\mathfrak{s p}_{4}$. Такая сцепка имеет сложность не ниже 4.

Этап 6. Аналогично этапу 4 проверяется, что у пар с $s=3$ нет валентных и 1-валентных компонент, отличных от $\mathfrak{s l}_{2}$.

Остается заметить, что для всех пар из таблицы 4 два различных выбора изоморфизма вдоль отрезка, определяюшего вложение компоненты подалгебры в компоненту алгебры, задают подалгебры, которые переводятся друг в друга автоморфизмом объемлюшей алгебры. Тем самым доказана

Теорема 3. Все неразложимые насыщенные пары $(\mathfrak{g}, \mathfrak{h})$ сложности один, где $\mathfrak{g}$ - полупростая алгебра Ли, а $\mathfrak{h}-$ ее редуктивная подалгебра, перечислены в табличе 4 с точностью до автоморфизма алгебры $\mathfrak{g}$.

Интересно отметить, что в таблице 4 нет пар четного ранга большего 8 , а нечетного ранга большего 7 имеется лишш одна серия - п. 27.

ЗАмЕчАниЕ 2. Покажем, как описать все неразложимыепары сложности один, если описаны все такие насыщенные пары. Идея излагаемого здесь метода была 
предложена Д. А. Тимашёвым. Каждой неразложимой паре $(\mathfrak{g}, \mathfrak{h})$ отвечает насыщенная (возможно, разложимая) пара $(\mathfrak{g}, \widetilde{\mathfrak{h}})$, где полупростые части $\mathfrak{h}$ и $\widetilde{\mathfrak{h}}$ совпадают (обозначим их $\mathfrak{h}^{s}$ ), а центр $\widetilde{\mathfrak{h}}$ (обозначим его $\widetilde{\mathfrak{z}}=\mathfrak{z}_{1} \oplus \cdots \oplus \mathfrak{z}_{s}$ ) является наименьшей вполне разложимой подалгеброй, содержашей центр $\mathfrak{h}$ (обозначим последний $\mathfrak{z})$. Если $c(\mathfrak{g}, \mathfrak{h})=1$, то $c(\mathfrak{g}, \widetilde{\mathfrak{h}}) \leqslant 1$. В алгебре $\widetilde{\mathfrak{z}}$ имеется наибольшая подалгебра $\mathfrak{p}$ такая, что

$$
c\left(\mathfrak{g}, \mathfrak{h}^{s}\right)=c\left(\mathfrak{g}, \mathfrak{h}^{s}+\mathfrak{p}\right)=c(\mathfrak{g}, \widetilde{\mathfrak{h}})-(\operatorname{dim} \widetilde{\mathfrak{z}}-\operatorname{dim} \mathfrak{p}) .
$$

В самом деле, подалгебра p является касательной алгеброй к максимальному подтору в центре групшы $\widetilde{H}$, который сохраняет типичные $H^{s}$-орбиты на $G / B$. Заметим, что $\mathfrak{p}$ разлагается в прямую сумму подалгебр в соответствии с разложением пары $(\mathfrak{g}, \widetilde{\mathfrak{h}})$ на неразложимые компоненты. Поэтому достаточно вычислить подалгебру $\mathfrak{p}$ для каждой насышенной неразложимой пары. Из таблиц 1-4 непосредственно следует, что подалгебра р нулевая во всех случаях, кроме

1) таблица 1: п. 2 при $n \neq m$; п. 6 ; п. 7 при $n$ нечетном; п. 25;

таблица $3:$ п. 1 при $n \geqslant 3$;

таблица $4:$ п. 8 при $n \geqslant 3, m \geqslant 3 ;$ п. 9 при $n \geqslant 3 ;$ п. 16 при $n \geqslant 3 ;$ п. 25 при $n \geqslant 4$, во всех этих случаях $\mathfrak{p}=\widetilde{\mathfrak{z}}$;

2) таблица 2, п. 2: здесь $\mathfrak{p}$ - одномерная подалгебра, описанная в п. 3;

3) таблица 4, п. 8 при $n=1,2, m \geqslant 3$ и $m=1,2, n \geqslant 3:$ здесь р одномерна и соответствует большей компоненте.

Остается заметить, что пара $(\mathfrak{g}, \mathfrak{h})$ имеет сложность 1 тогда и только тогда, когда либо $c(\mathfrak{g}, \widetilde{\mathfrak{h}})=1$ и подалгебра $\mathfrak{z}$ при ограничении на нее проекции $\widetilde{\mathfrak{z}} \rightarrow \widetilde{\mathfrak{z}} / \mathfrak{p}$ отображается на образ сюръективно, либо $c(\mathfrak{g}, \widetilde{\mathfrak{h}})=0$ и образ $\mathfrak{z}$ при указанной проекции является гиперплоскостью.

Обозначения и соглашения, использованные в таблицах. Индексы $n, m$ в таблицах 1 и 2 предполагаются бо́льшими или равными 1 , а индексы $n, m, k, l$ в таблицах 3 и 4 предполагаются бо́льшими или равными 0 , если не оговорено противное. Если индекс классической алгебры принимает неположительное значение (например, $\mathfrak{s p}_{2 n-2}$ при $n=0,1$ ), соответствующая алгебра считается нулевой. Символ Кронекера $\delta_{i}^{j}$ равен 1 при $i=j$ и 0 в противном случае. В таблицах 1 и 2 столбец " $\mathfrak{h} \subset \mathfrak{g}$ " определяет вложение подалгебры $\mathfrak{h}$ в алгебру $\mathfrak{g}$ посредством описания ограничения на $\mathfrak{h}$ первого фундаментального представления алгебры g. (Нумерация фундаментальных весов простых алгебр Ли соответствует нумерации из [21].)

Символами $\pi_{i}$ и $\pi_{i}^{\prime}$ обозначены фундаментальные веса первой и второй простых компонент алгебры $\mathfrak{h}$ соответственно. Символ $\varepsilon$ обозначает фундаментальный вес одномерной центральной подалгебры $\mathfrak{c}$. Для весов использована мультипликативная запись, т.е. $\pi_{1}^{2}$ - это старший вес, у которого первая отметка равна 2 , а прочие отметки нулевые. Знак “+” обозначает прямую сумму представлений. Символом 1 обозначено одномерное тривиальное представление, символом 2 - двумерное тривиальное и т.д. В таблицах 3 и 4 вложение подалгебры в алгебру обозначено отрезками. Если отрезок может быть неоднозначно истолкован, вложение обозначено на отрезке, подобно таблицам 1 и 2. 
ТАБЛИЦА 1

\begin{tabular}{|c|c|c|c|c|}
\hline & $\mathfrak{g}$ & $\mathfrak{h}$ & $\mathfrak{h} \subset \mathfrak{g}$ & $\mathfrak{m}$ \\
\hline 1 & $\mathfrak{s l}_{n}, n \geqslant 2$ & $\mathfrak{s o}_{n}$ & $\pi_{1}$ & $\pi_{1}^{2}, n \neq 2,4$ \\
\hline 2 & $\mathfrak{s l}_{n+m}$ & $\mathfrak{s l}_{n} \oplus \mathfrak{s l}_{m} \oplus \mathfrak{c}$ & $\pi_{1} \varepsilon^{m}+\pi_{1}^{\prime} \varepsilon^{-n}$ & $\begin{array}{l}\pi_{1} \otimes \pi_{m-1}^{\prime} \varepsilon^{m+n} \\
\quad+\pi_{n-1} \otimes \pi_{1}^{\prime} \varepsilon^{-m-n}\end{array}$ \\
\hline 3 & $\mathfrak{s l}_{n+m}, m \neq n$ & $\mathfrak{s l}_{n} \oplus \mathfrak{s l}_{m}$ & $\pi_{1}+\pi_{1}^{\prime}$ & $\pi_{1} \otimes \pi_{m-1}^{\prime}+\pi_{n-1} \otimes \pi_{1}^{\prime}+1$ \\
\hline 4 & $\mathfrak{s l}_{2 n}, n \geqslant 2$ & $\mathfrak{s p}_{2 n}$ & $\pi_{1}$ & $\pi_{2}$ \\
\hline 5 & $\mathfrak{s l}_{2 n+1}$ & $\mathfrak{s p}_{2 n}$ & $\pi_{1}+1$ & $\pi_{1}^{2}+\pi_{2}+1$ \\
\hline 6 & $\mathfrak{s l}_{2 n+1}$ & $\mathfrak{s p}_{2 n} \oplus \mathfrak{c}$ & $\pi_{1} \varepsilon+\varepsilon^{-2 n}$ & $\pi_{1} \varepsilon^{2 n+1}+\pi_{1} \varepsilon^{-2 n-1}+\pi_{2}$ \\
\hline 7 & $\mathfrak{s o}_{2 n}$ & $\mathfrak{s l}_{n} \oplus \mathfrak{c}$ & $\pi_{1} \varepsilon+\pi_{n-1} \varepsilon^{-1}$ & $\pi_{2} \varepsilon^{2}+\pi_{n-2} \varepsilon^{-2}$ \\
\hline 8 & $\mathfrak{s o}_{4 n+2}$ & $\mathfrak{s l}_{2 n+1}$ & $\pi_{1}+\pi_{2 n}$ & $\pi_{2}+\pi_{2 n-1}+1$ \\
\hline 9 & $\mathfrak{s o}_{2 n+1}$ & $\mathfrak{s l}_{n} \oplus \mathfrak{c}$ & $\pi_{1} \varepsilon+\pi_{n-1} \varepsilon^{-1}+1$ & $\begin{array}{l}\pi_{1} \varepsilon+\pi_{2} \varepsilon^{2}+\pi_{n-2} \varepsilon^{-2} \\
\quad+\pi_{n-1} \varepsilon^{-1}\end{array}$ \\
\hline 10 & $\mathfrak{s o}_{n+m}$ & $\mathfrak{s o}_{n} \oplus \mathfrak{s o}_{m}$ & $\pi_{1}+\pi_{1}^{\prime}$ & $\pi_{1} \otimes \pi_{1}^{\prime}$ \\
\hline 11 & $\mathfrak{s o g}_{9}$ & $\mathfrak{s o}_{7}$ & $\pi_{3}+1$ & $\pi_{1}+\pi_{3}$ \\
\hline 12 & $\mathfrak{s o}_{7}$ & $G_{2}$ & $\pi_{1}$ & $\pi_{1}$ \\
\hline 13 & $\mathfrak{s o}_{8}$ & $G_{2}$ & $\pi_{1}+1$ & $\pi_{1}^{2}$ \\
\hline 14 & $\mathfrak{s o}_{10}$ & $\mathfrak{s o}_{7} \oplus \mathfrak{c}$ & $\pi_{3}+\varepsilon+\varepsilon^{-1}$ & $\pi_{1}+\pi_{3} \varepsilon+\pi_{3} \varepsilon^{-1}$ \\
\hline 15 & $\mathfrak{s p}_{2 n}$ & $\mathfrak{s l}_{n} \oplus \mathfrak{c}$ & $\pi_{1} \varepsilon+\pi_{n-1} \varepsilon^{-1}$ & $\pi_{1}^{2} \varepsilon^{2}+\pi_{n-1}^{2} \varepsilon^{-2}$ \\
\hline 16 & $\mathfrak{s p}_{2(n+m)}$ & $\mathfrak{s p}_{2 n} \oplus \mathfrak{s p}_{2 m}$ & $\pi_{1}+\pi_{1}^{\prime}$ & $\pi_{1} \otimes \pi_{1}^{\prime}$ \\
\hline 17 & $\mathfrak{s p}_{2 n}$ & $\mathfrak{s p}_{2(n-1)} \oplus \mathfrak{c}$ & $\pi_{1}+\varepsilon+\varepsilon^{-1}$ & $\pi_{1} \varepsilon+\pi_{1} \varepsilon^{-1}+\varepsilon^{2}+\varepsilon^{-2}$ \\
\hline 18 & $G_{2}$ & $\mathfrak{s l}_{3}$ & $\pi_{1}+\pi_{2}+1$ & $\pi_{1}+\pi_{2}$ \\
\hline 19 & $G_{2}$ & $\mathfrak{s l}_{2} \oplus \mathfrak{s l}_{2}$ & $\pi_{1}^{2}+\pi_{1} \otimes \pi_{1}^{\prime}$ & $\pi_{1}^{3} \otimes \pi_{1}^{\prime}$ \\
\hline 20 & $F_{4}$ & $\mathfrak{s o g}$ & $\pi_{1}+\pi_{4}+1$ & $\pi_{4}$ \\
\hline 21 & $F_{4}$ & $\mathfrak{s p}_{6} \oplus \mathfrak{s l}_{2}$ & $\pi_{2}+\pi_{1} \otimes \pi_{1}^{\prime}$ & $\pi_{3} \otimes \pi_{1}^{\prime}$ \\
\hline 22 & $E_{6}$ & $\mathfrak{s p}_{8}$ & $\pi_{2}$ & $\pi_{4}$ \\
\hline 23 & $E_{6}$ & $F_{4}$ & $\pi_{1}+1$ & $\pi_{1}$ \\
\hline 24 & $E_{6}$ & $\mathfrak{s o}_{10}$ & $\pi_{1}+\pi_{5}+1$ & $\pi_{4}+\pi_{5}+1$ \\
\hline 25 & $E_{6}$ & $\mathfrak{s o}_{10} \oplus \mathfrak{c}$ & $\pi_{1} \varepsilon^{2}+\pi_{5} \varepsilon^{-1}+\varepsilon^{-4}$ & $\pi_{4} \varepsilon^{6}+\pi_{5} \varepsilon^{-6}$ \\
\hline 26 & $E_{6}$ & $\mathfrak{s l}_{6} \oplus \mathfrak{s l}_{2}$ & $\pi_{4}+\pi_{1} \otimes \pi_{1}^{\prime}$ & $\pi_{3} \otimes \pi_{1}^{\prime}$ \\
\hline 27 & $E_{7}$ & $E_{6} \oplus \mathfrak{c}$ & $\begin{array}{l}\pi_{1} \varepsilon+\pi_{5} \varepsilon^{-1} \\
\quad+\varepsilon^{3}+\varepsilon^{-3}\end{array}$ & $\pi_{1} \varepsilon^{4}+\pi_{5} \varepsilon^{-4}$ \\
\hline 28 & $E_{7}$ & $\mathfrak{s l}_{8}$ & $\pi_{2}+\pi_{6}$ & $\pi_{4}$ \\
\hline
\end{tabular}


ТАБлицА 1 (продолжение)

\begin{tabular}{|c|c|c|c|c|}
\hline & $\mathfrak{g}$ & $\mathfrak{h}$ & $\mathfrak{h} \subset \mathfrak{g}$ & $\mathfrak{m}$ \\
\hline 29 & $E_{7}$ & $\mathfrak{s o}_{12} \times \mathfrak{s l}_{2}$ & $\pi_{6}+\pi_{1} \otimes \pi_{1}^{\prime}$ & $\pi_{5} \otimes \pi_{1}^{\prime}$ \\
\hline 30 & $E_{8}$ & $\mathfrak{s o}_{16}$ & $\pi_{2}+\pi_{8}$ & $\pi_{7}$ \\
\hline 31 & $E_{8}$ & $\mathfrak{s l}_{2} \times E_{7}$ & $\pi_{1} \otimes \pi_{1}^{\prime}+\pi_{1}^{2}+\pi_{6}^{\prime}$ & $\pi_{1} \otimes \pi_{1}^{\prime}$ \\
\hline
\end{tabular}

ТАБЛИЦА 2

\begin{tabular}{|c|c|c|c|c|}
\hline & $\mathfrak{g}$ & $\mathfrak{h}$ & $\mathfrak{h} \subset \mathfrak{g}$ & $\mathfrak{m}$ \\
\hline 1 & $\mathfrak{s l}_{2 n}$ & $\mathfrak{s l}_{n} \oplus \mathfrak{s l}_{n}$ & $\pi_{1}+\pi_{1}^{\prime}$ & $\begin{array}{l}\pi_{1} \otimes \pi_{1}^{\prime} \\
\quad+\pi_{n-1} \otimes \pi_{n-1}^{\prime}+1\end{array}$ \\
\hline 2 & $\begin{array}{c}\mathfrak{s l}_{n} \\
n \geqslant 3\end{array}$ & $\mathfrak{s l}_{n-2} \oplus \mathfrak{c} \oplus \mathfrak{c}$ & $\pi_{1} \varepsilon_{1} \varepsilon_{2}+\varepsilon_{1}^{2-n}+\varepsilon_{2}^{2-n}$ & $\begin{array}{l}\pi_{1} \varepsilon_{1}^{n-1} \varepsilon_{2}+\pi_{1} \varepsilon_{1} \varepsilon_{2}^{n-1} \\
\quad+\varepsilon_{1}^{2-n} \varepsilon_{2}^{n-2} \\
\quad+\varepsilon_{1}^{n-2} \varepsilon_{2}^{2-n} \\
\quad+\pi_{n-3} \varepsilon_{1}^{1-n} \varepsilon_{2}^{-1} \\
\quad+\pi_{n-3} \varepsilon_{1}^{-1} \varepsilon_{2}^{1-n}\end{array}$ \\
\hline 3 & $\begin{array}{c}\mathfrak{s l}_{n} \\
n \geqslant 5\end{array}$ & $\mathfrak{s l}_{n-2} \oplus \mathfrak{c}$ & $\begin{array}{c}\pi_{1} \varepsilon+\varepsilon^{d_{1}}+\varepsilon^{d_{2}} \\
d_{1} \neq d_{2} \\
d_{1}+d_{2}=2-n\end{array}$ & $\begin{array}{l}\pi_{1} \varepsilon^{1-d_{1}}+\pi_{1} \varepsilon^{1-d_{2}} \\
\quad+\varepsilon^{d_{1}-d_{2}}+\varepsilon^{d_{2}-d_{1}} \\
\quad+\pi_{n-3} \varepsilon^{d_{1}-1} \\
\quad+\pi_{n-3} \varepsilon^{d_{2}-1}\end{array}$ \\
\hline 4 & $\mathfrak{s l}_{6}$ & $\mathfrak{s p}_{4} \oplus \mathfrak{s l}_{2} \oplus \mathfrak{c}$ & $\pi_{1} \varepsilon+\pi_{1}^{\prime} \varepsilon^{-2}$ & $\begin{array}{l}\pi_{1} \otimes \pi_{1}^{\prime} \varepsilon+\pi_{2} \\
\quad+\pi_{1} \otimes \pi_{1}^{\prime} \varepsilon^{-1}\end{array}$ \\
\hline 5 & $\begin{array}{c}\mathfrak{s o} n \\
n \geqslant 5\end{array}$ & $\mathfrak{s o}_{n-2}$ & $\pi_{1}+2$ & $\pi_{1}+\pi_{1}+1$ \\
\hline 6 & $\begin{array}{l}\mathfrak{s o}_{2 n+1} \\
n \geqslant 3\end{array}$ & $\mathfrak{s l}_{n}$ & $\pi_{1}+\pi_{n-1}+1$ & $\begin{array}{c}\pi_{1}+\pi_{2}+\pi_{n-1} \\
\quad+\pi_{n-2}+1\end{array}$ \\
\hline 7 & $\begin{array}{l}\mathfrak{s o}_{4 n} \\
n \geqslant 2\end{array}$ & $\mathfrak{s l}_{2 n}$ & $\pi_{1}+\pi_{2 n-1}$ & $\pi_{2}+\pi_{2 n-2}$ \\
\hline 8 & $\mathfrak{s o g}_{9}$ & $G_{2} \oplus \mathfrak{c}$ & $\pi_{1}+\varepsilon+\varepsilon^{-1}$ & $\pi_{1}+\pi_{1} \varepsilon+\pi_{1} \varepsilon^{-1}$ \\
\hline 9 & $\mathfrak{s o}_{11}$ & $\mathfrak{s l}_{2} \oplus \mathfrak{s o}_{7}$ & $\pi_{1}^{2}+\pi_{3}^{\prime}$ & $\pi_{1}^{2} \otimes \pi_{3}^{\prime}+\pi_{1}^{\prime}$ \\
\hline 10 & $\mathfrak{s o}_{10}$ & $\mathfrak{s o}_{7}$ & $\pi_{3}+2$ & $\pi_{1}+\pi_{3}+\pi_{3}+1$ \\
\hline 11 & $\mathfrak{s p}_{2 n}$ & $\mathfrak{s l}_{n}$ & $\pi_{1}+\pi_{n-1}$ & $\pi_{1}^{2}+\pi_{n-1}^{2}+1$ \\
\hline 12 & $\begin{array}{l}\mathfrak{s p}_{2 n} \\
n \geqslant 2\end{array}$ & $\mathfrak{s p}_{2 n-2}$ & $\pi_{1}+2$ & $\pi_{1}+\pi_{1}+3$ \\
\hline 13 & $\begin{array}{l}\mathfrak{s p}_{2 n} \\
n \geqslant 3\end{array}$ & $\mathfrak{s p}_{2 n-4} \oplus \mathfrak{s l}_{2} \oplus \mathfrak{s l}_{2}$ & $\pi_{1}+\pi_{1}^{\prime}+\pi_{1}^{\prime \prime}$ & $\begin{aligned} \pi_{1} \otimes & \pi_{1}^{\prime}+\pi_{1} \otimes \pi_{1}^{\prime \prime} \\
& +\pi_{1}^{\prime} \otimes \pi_{1}^{\prime \prime}\end{aligned}$ \\
\hline 14 & $\mathfrak{s p}_{4}$ & $\mathfrak{s l}_{2}$ & $\pi_{1}^{3}$ & $\pi_{1}^{6}$ \\
\hline 15 & $E_{6}$ & $\mathfrak{s o}_{9} \oplus \mathfrak{c}$ & $\pi_{1} \varepsilon^{2}+\varepsilon^{2}+\pi_{4} \varepsilon^{-1}+\varepsilon^{-4}$ & $\pi_{1}+\pi_{4} \varepsilon+\pi_{4} \varepsilon^{-1}$ \\
\hline 16 & $E_{7}$ & $E_{6}$ & $\pi_{1}+\pi_{5}+2$ & $\pi_{1}+\pi_{5}+1$ \\
\hline 17 & $F_{4}$ & $\mathfrak{s o}_{8}$ & $\pi_{1}+\pi_{3}+\pi_{4}+2$ & $\pi_{1}+\pi_{3}+\pi_{4}$ \\
\hline
\end{tabular}


ТАБЛИЦА 3

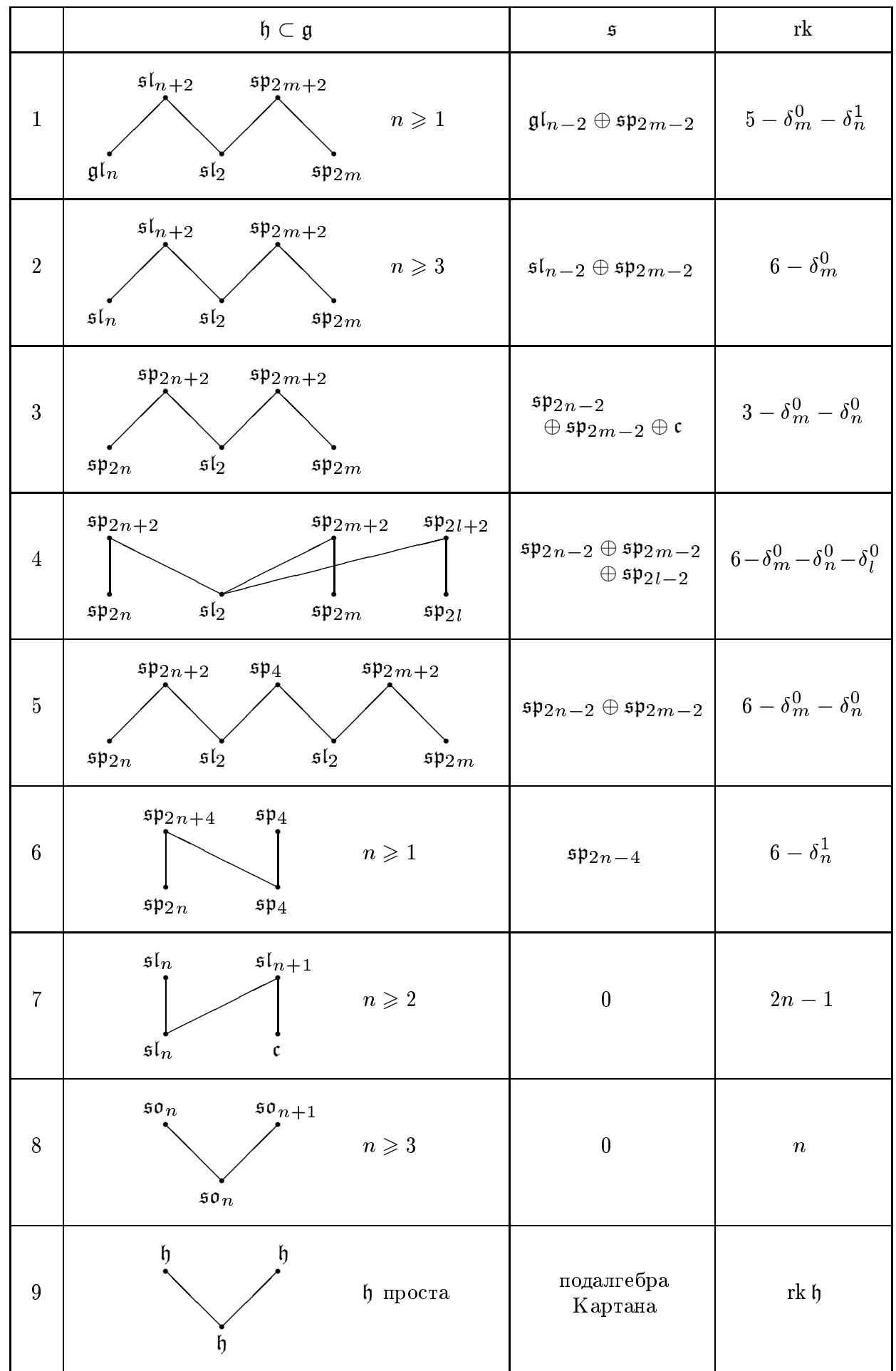


ТАБЛИЦА 4

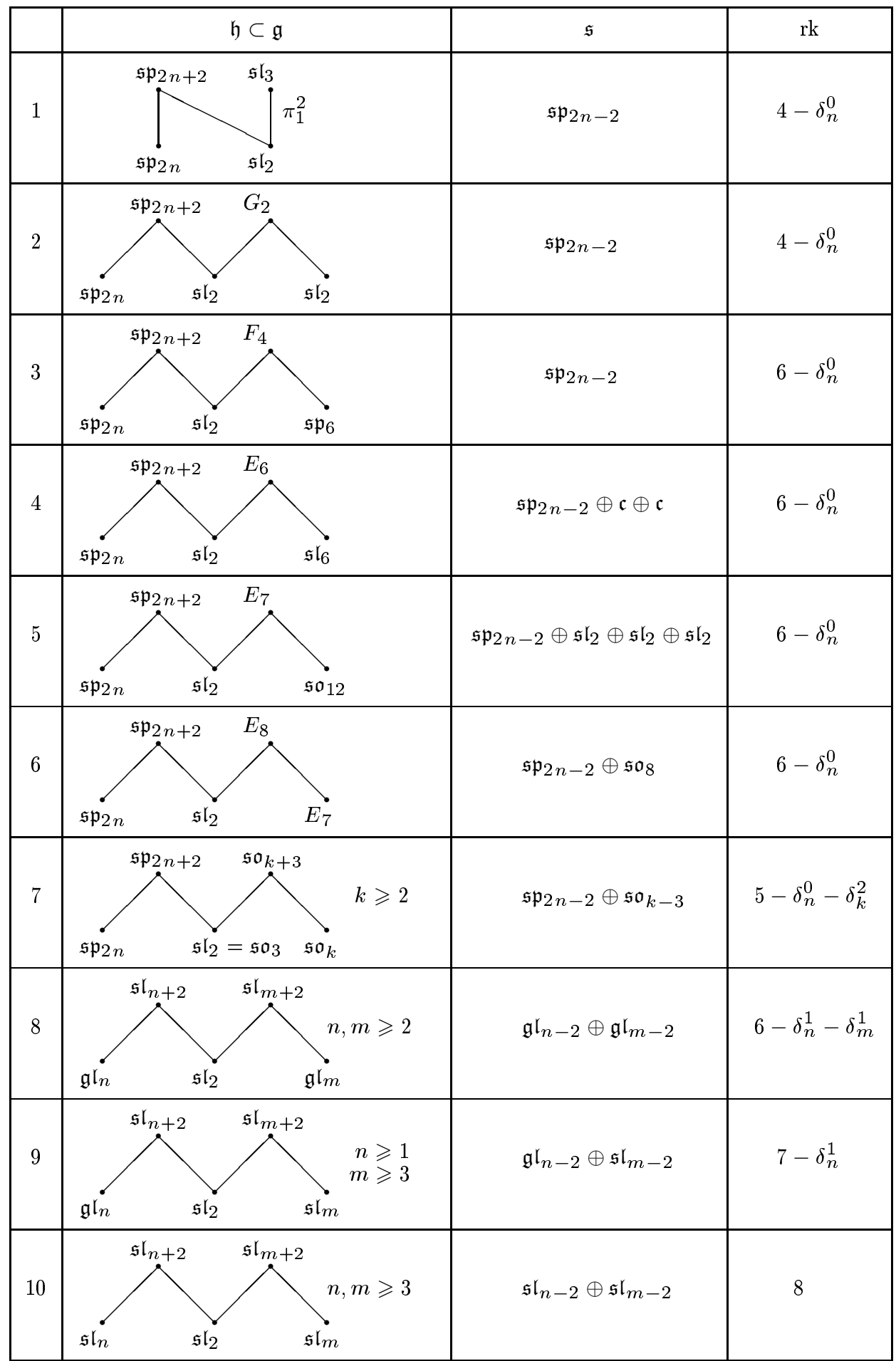


ТАБлицА 4 (продолжение)

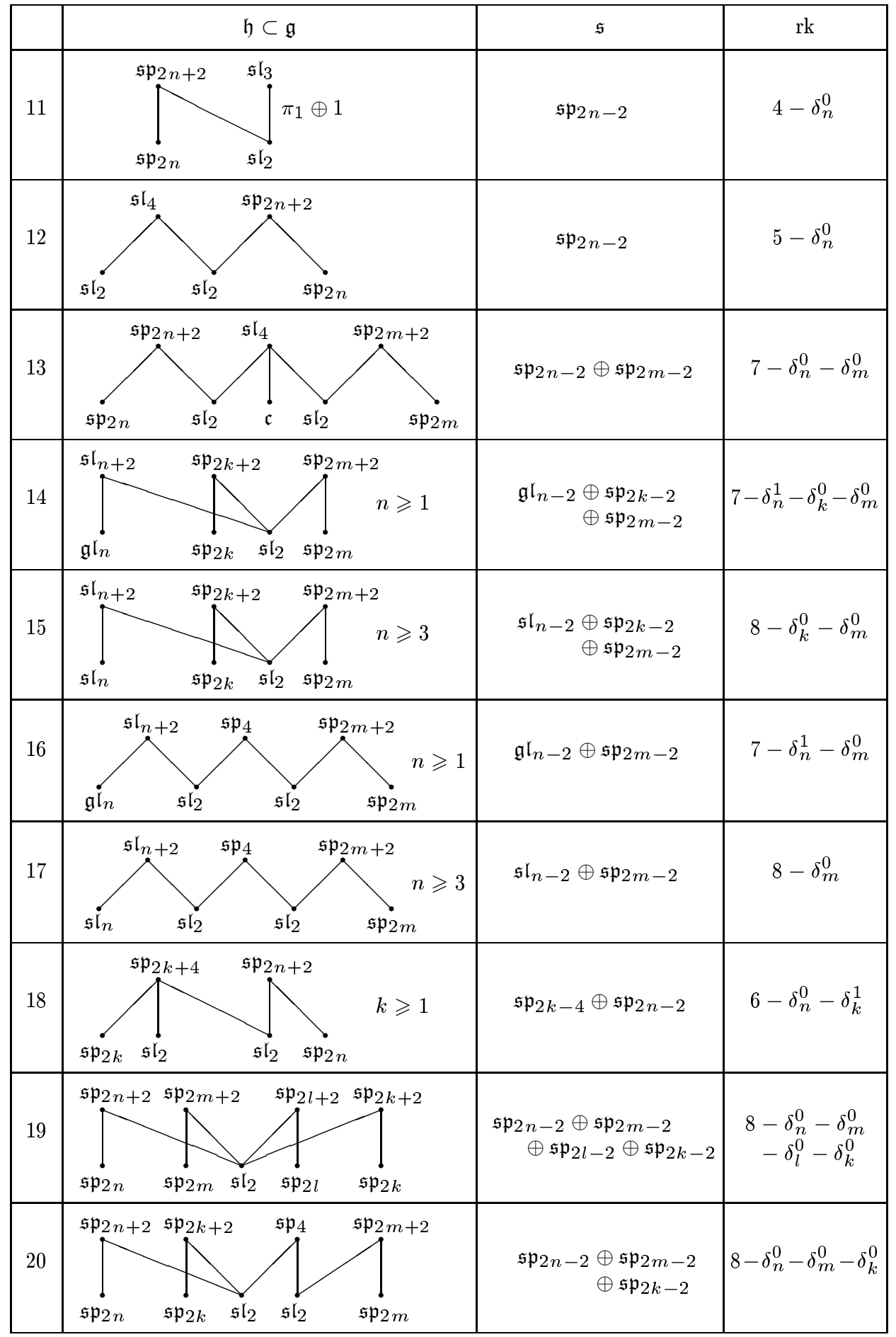


ТАБлицА 4 (продолжение)

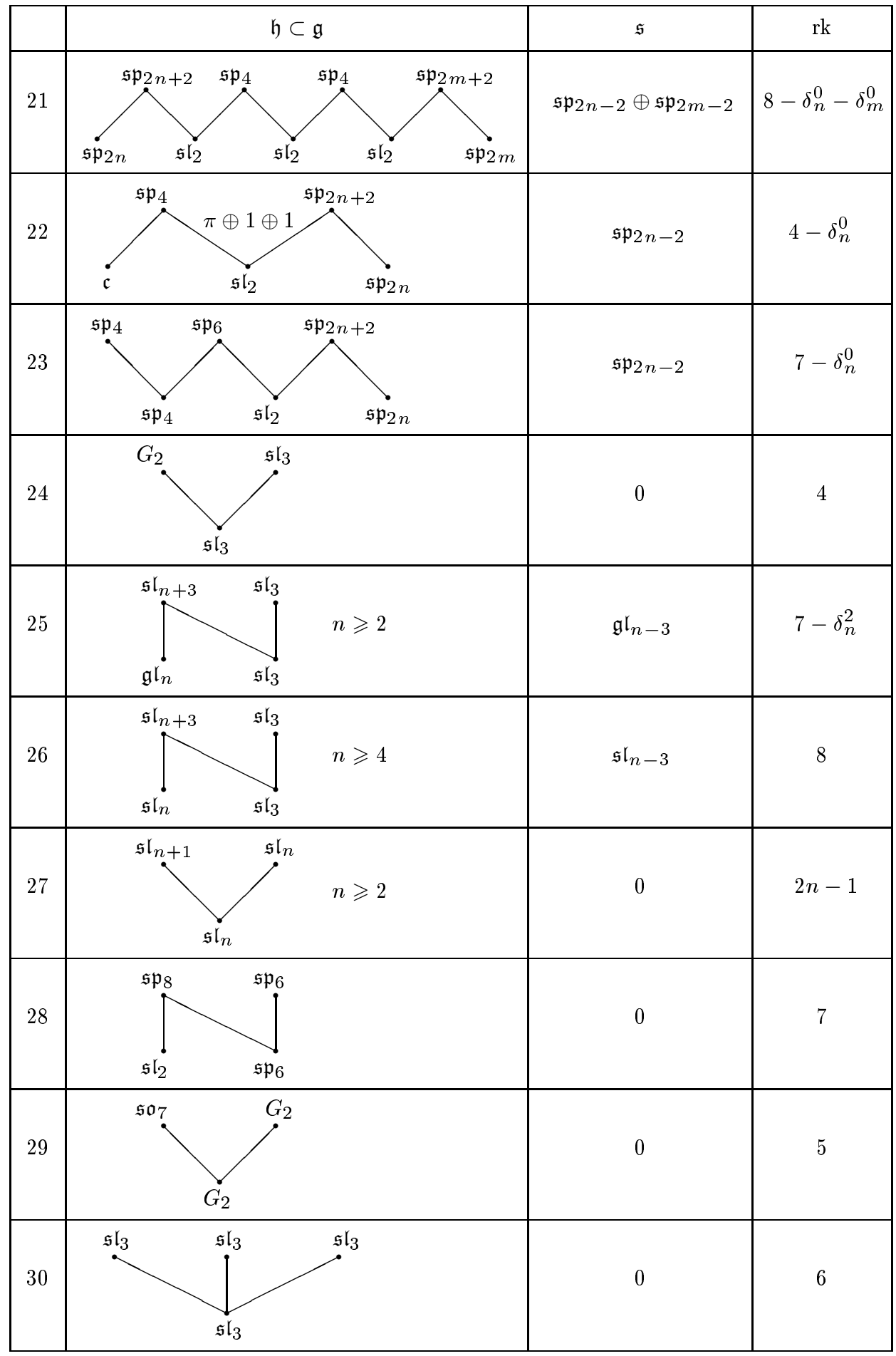




\section{Список литературы}

1. Luna D., Vust Th. Plongements d'espaces homogènes // Comment. Math. Helv. 1983. V. 58. P. $186-245$.

2. Винберг Э. Б. Сложность действий редуктивных групп // Функц. анализ и его прилож. 1986. T. 20. № 1. C. 1-13.

3. Brion M. Variétés sphériques // Notes de la session de la S.M.F. "Opérations hamiltoniennes et opérations de groupes algébriques". Grenoble, 1997; http://www-fourier.ujfgrenoble.fr/ “mbrion/spheriques.ps.

4. Knop F. The Luna-Vust theory of spherical embeddings // Proceeding of the Hyderabad conference on algebraic groups. (Univ. Hyderabad, India, December 1989) / ed. S. Ramanan. Madras: Manoj Prakashan, 1991. P. 225-249.

5. Тимашев Д. А. Классификация $G$-многообразий сложности 1 // Изв. РАН. Сер. матем. 1997. Т. 61. № 2. С. 127-162.

6. Ахиезер Д. Н. О действиях с конечнњм числом орбит // Функц. анализ и его прилож. 1985. Т. 19. № 1. С. 1-5.

7. Ахиезер Д. Н. О модальности и сложности действий редуктивных групп // УМН. 1988. T. 43. № 2. C. 129-130.

8. Arzhantsev I. V., Timashev D.A. Affine embeddings with a finite number of orbits // Transform. Groups. 2001. V. 6. № 2. P. 101-110.

9. Аржанцев И. В. О модальности и сложности афффинных вложений // Матем. сб. 2001. T. 192. № 8. C. $47-52$.

10. Микитюк И. В. Об интегрируемости инвариантных гамильтоновых систем с однородными конфигурационньми пространствами // Матем. сб. 1986. Т. 129 (171). С. 514-534.

11. Gullemin V., Sternberg S. Multiplicity-free spaces // J. Differential Geom. 1984. V. 19. P. $31-56$.

12. Винберг Э. Б. Коммутативные однородные пространства и коизотропные симплектические действия // УМН. 2001. Т. 56. №1. С. 3-62.

13. Mykytyuk I. V., Stepin A. M. Classification of almost spherical pairs of compact simple Lie groups // Poisson geometry / ed. J. Grabowski. Warsawa: Polish Acad. Sci., Inst. Math., 2000. P. 231-241. (Banach Center Publ. V. 51.)

14. Mykytyuk I. V. Actions of Borel subgroups on homogeneous spaces of reductive complex Lie groups and integrability // Compositio Math. 2001. V. 127. P. 55-67.

15. Krämer $M$. Sphärische Untergruppen in kompacten zusammenhängenden Liegruppen // Compositio Math. 1979. T. 38. C. 129-153.

16. Brion M. Classification des espaces homogènes sphériques // Compositio Math. 1987. V. 63. P. 189-208.

17. Panyushev D.I. Complexity of quasiaffine homogeneous varieties, $t$-decompositions, and affine homogeneous spaces of complexity $1 / /$ Adv. Soviet Math. 1992. V. 8. P. 151-166.

18. Элашвили А. Г. Канонический вид и стационарныеподалгебры точек общего положения для простых линейных групп Ли // Функц. анализ и его прилож. 1972. Т. 6. № 1. С. 51-62.

19. Panyushev D. I. Complexity and rank of homogeneous spaces // Geom. Dedicata. 1990. V. 34. P. 249-269.

20. Panyushev D. I. Complexity and nilpotent orbits // Manuscripta Math. 1994. V. 83. P. 223-237.

21. Винберг Э. Б., Онищик А. Л. Семинар по группам Ли и алгебраическим группам. М.: Наука, 1988.

Московский государственнњй университет

Поступила в редакцию

им. М.В. Ломоносова

06.03.2003 и 12.01.2004

E-mail: arjantse@mccme.ru

chuvashova@yandex.ru 